\title{
Biopoder: el poder y la violencia en la formación de profesionales de enfermería
}

\author{
R. Ayala-Valenzuela
}

\begin{abstract}
El presente artículo da cuenta del ejercicio del poder en las relaciones existentes entre los profesionales de salud en formación (estudiantes) y sus preceptores (profesionales de enfermería); el modo en que éstos - desde su posición asimétrica de poder-generan sentimientos adversos para el aprendizaje de aquéllos, a través de la violencia simbólica y la violencia explícita, en el afán de controlar el cuerpo en términos de su productividad y su rendimiento. Fundamenta sus argumentaciones a partir de las ideas de Pierre Bordieu desde la escuela estructuralista, y las de Michael Foucault y Gilles Deleuze desde la perspectiva postestructuralista, quienes hicieron sus aportaciones a las ideas de control, poder, biopoder y violencia simbólica. Hace una analogía de esta situación de maltrato hacia estudiantes con los círculos que se generan en escenarios de violencia intrafamiliar, reconociendo roles homólogos en ambos contextos: la víctima, el victimario, los testigos silentes y los testigos activos. Por último, relaciona estos razonamientos con estudios de percepción de la violencia en estudiantes de enfermería en los campos clínicos chilenos.
\end{abstract}

Palabras clave. Biopoder. Enfermería. Formación.

\section{Bio-power: power and violence \\ in the education of nurses}

The present article is an account of the power exercise in the existing relations between the professionals of health in formation (students) and its teachers (nurses); how teachers, from an asymmetric power position, generate adverse feelings for the learning of those, through the symbolic violence and the explicit violence, in the eagerness to control the body in terms of its productivity and its yield. It bases its argumentations from the ideas of Pierre Bordieu from the structuralist school, and those of Michael Foucault and
Gilles Deleuze from the post-structuralist perspective, who made their contributions to the ideas of control, power, biopower and symbolic violence. It makes an analogy of this situation of mistreat towards students with the circles that are generated in domestic violence, recognizing homologous rolls in both contexts: the victim, the aggressor, the silent witnesses and the active witnesses. Finally, it relates these reasoning to studies of perception of violence towards the students of nursing in Chilean clinical teaching.

Key words. Bio-power. Nursing. Teaching.

\section{Enfermería y poder-género-violencia}

La enfermería como profesión se ha desarrollado apenas desde mediados del siglo XIX, y se encuentra estrechamente relacionada con el concepto victoriano que se tiene del rol de la mujer en la sociedad. Así pues, en su definición del rol de la enfermería, Florence Nightingale subraya la dependencia y subordinación de las enfermeras (mujeres) respecto a los médicos (varones) [1], naciendo supeditada por las relaciones de poder inmanente en las relaciones de género. Sin embargo, se habla del nacimiento de una violencia horizontal (secundaria), a partir de las relaciones de poder-género (primaria) al analizar el comportamiento de la enfermería en la lógica de los grupos oprimidos. 'Dentro de esta perspectiva, los valores y normas del grupo dominante (en este caso, la medicina) dan por resultado poder y control; la incapacidad de rebelarse en contra del grupo que ejerce el poder conduce a un 'síndrome de agresión su-
Facultad de Medicina. Instituto de Enfermería. Universidad Austral de Chile. Valdivia, Chile.

Correspondencia Prof. Ricardo Ayala Valenzuela. Facultad de Medicina. Instituto de Enfermería. Universidad Austral de Chile. Isla Teja, s/n. Valdivia, Chile.

E-mail

ricardoayala@uach.cl

Extracto del trabajo presentado en la cátedra del Dr. Browne Sartori Comunicación y poder, de la Facultad de Filosofía y Humanidades, Universidad Austral de Chile. 
misa'... lo cual tiene como resultado una 'violencia horizontal' manifestada por conflictos internos y temor al cambio. El liderazgo en el grupo menos poderoso se caracteriza por 'odio a su propia especie', deseo de ser como el opresor, y comportamiento rígido, determinante y coercitivo' [2]. Estas palabras sin duda ilustran en gran medida los serios problemas que la profesión enfermera ha tenido en su corta existencia, tanto para definirse a sí misma como en el modo de crear ambientes de desarrollo y formación de semejantes. Entre todas estas tensiones de identidad, de rol, de posición, aparece el actor 'profesional en formación': el estudiante, en el lenguaje usual, quien va a ser, como se explica más adelante, víctima de los ejercicios de poder y de violencia concreta y simbólica, primaria o secundaria.

\section{Análisis del poder en su transversalidad}

El poder no tiene manifestaciones únicas; tiene, por el contrario, una extensa gama de forma y naturaleza, entendiéndolo en su omnipresencia en las relaciones humanas, de padre e hijo, de hombre y mujer, de profesor y estudiante, de médico y enfermo, de hermano mayor y hermano menor, de policía y civil, de ricos y pobres, de instruidos e ignorantes. Hablamos, pues, de 'las múltiples formas de dominación que pueden ejercerse en el interior de la sociedad' [3], en los establecimientos educacionales de todo tipo (lugar preferido para reproducir relaciones de poder), en el hospital, en las iglesias, en la cárcel; todos ellos constituyen espacios básicos en los que se definen situaciones de poder. Las grandes dimensiones donde se agrupan expresiones heterogéneas del poder difieren en sus dinámicas, pero desarrollan formas de perpetuarse a través de mecanismos de consolidación y de legitimación a partir de los cuales elaboran su discurso. La primera dimensión está determinada por las relaciones interpersonales entre los miembros de una colectividad. La segunda, por las formas institucionalizadas que operan como espacios cerrados. Así pues, el poder trasciende en sus expresiones más allá de la de un individuo por encima de otro: la de un grupo sobre otro, los miembros del cual permanecen como presa de sus condiciones para el ejercicio de ese poder. Nos acompaña durante toda nuestra vida, por todos los espacios de encierro en los que nos movemos, regidos ellos por sus propios sistemas de valores: 'primero la familia; enseguida, la escuela ('ya no estás en tu familia'); luego el cuartel ('ya no estás en la escuela'); luego la fábrica; de vez en cuando el hospital y, acaso, alguna vez la prisión' [4].

\section{La disciplina y el control de los cuerpos (biopoder)}

Cobra énfasis el control y la manipulación intencionada del cuerpo como hombre-máquina, 'al que se da forma, se educa, obedece, responde, se vuelve hábil o cuyas fuerzas se multiplican' [5]. El cuerpo fue materia de estudio de la época clásica en el sentido de conocerlo, comprenderlo, de hacerlo inteligible. Muy por el contrario, en un aspecto político y técnico, lo situamos hoy en planos de sometimiento a través de normas militares, escolares y hospitalarias para controlar o corregir las operaciones del cuerpo [5]. La educación ejerce un rol muy selecto en este modelaje, ejerciendo su disciplina bajo coerción ininterrumpida. Estas inquebrantables limitaciones esculpen los movimientos en la dimensión espacio-tiempo, garantizando la contención de sus fuerzas y la utilidad mercantil de los cuerpos dóciles, siendo capaz de prescindir de la violencia (de la violencia concreta, al menos), pero cargada de simbolismos violentos, con beneficios directamente proporcionales a la docilidad de los cuerpos. Así, los cuerpos entran en una maraña de poderes sobreentendidos para que hagan lo que se desea, que 'operen como se quiere, con las técnicas, según la rapidez y la eficacia que se determina' [5].

'El control disciplinario no consiste simplemente en enseñar o en imponer una serie de gestos definidos; impone la mejor relación entre un gesto y la actitud global del cuerpo, que es su condición de eficacia y de rapidez' [5]. Según Matejski (citado en [1]), 'La enfermera es un soldado. La obediencia absoluta e incondicional es la idea fundamental del sistema militar... La rigidez y la exactitud producen mejores enfermeras'. 


\section{Violencia y violencia simbólica}

Es difícil poder hablar de la violencia como un vocablo que sea entendido por todas las personas como una misma cosa. Con el interés de representar la violencia dirigida a los estudiantes de carreras sanitarias, utilizaremos la siguiente definición: 'Violencia es aquella fuerza orientada y selectiva ejercida contra alguien provocándole daño, profanación, ofensa, deshonra, quebranto, o tomándolo como objeto de nuestra ira apelando al vigor o a la energía, y teniendo presente que no hace falta un golpe de puño o una patada, sino que un grito, una actitud de desprecio o una velada discriminación, por el motivo que fuere' [6].

En el contexto de la educación universitaria, la violencia no procede primordialmente de entre los estudiantes, sino que lo hace de los propios preceptores, a través de gestos, miradas, omisiones, actos discriminatorios, entonación de la voz y uso del modo imperativo en las formas verbales ('anda', 'apúrate', 'trae', 'ven', 'haz', 'escribe'). Desde la filosofía se describe el poder simbólico como aquél que es invisible, pero que existe física y objetivamente y que se ejerce con la complicidad de quien lo padece. Quizá sea este último punto la piedra angular de la violencia simbólica, al reconocerle como una forma válida de relación humana. La violencia simbólica es la que insta a imponer significaciones y que está aceptada implícitamente en un determinado ámbito social, debido a su propia carga de invisibilidad y simbolismo que la hace aún más perjudicial' [7].

\section{Violencia dirigida a los estudiantes de enfermería}

En Chile, destacan los estudios hechos por Letelier y Valenzuela [8] en los que se caracteriza al 'hospital' como un 'centro educacional' para los estudiantes de enfermería, puesto que desarrollan gran parte de su formación en contextos clínicos, pero que 'en muchas ocasiones no poseen un clima ideal para dicho fin'. Cuantificaron que más de un $80 \%$ de los estudiantes de enfermería perciben algún grado de violencia, y que prácticamente un 95\% reconoce algún tipo de violencia ejercida hacia sus compañeros. Lo que más destaca es que identifican como principal agente de violencia a las enfermeras. Nos encontramos, pues, ante la gran paradoja de esta disciplina, pues se define a sí misma como la 'gestión del cuidado'. Estos malos tratos quizá se expliquen, en parte, por lo expuesto antes, sobre el 'síndrome de la agresión sumisa', en el que las enfermeras dirigen actos de violencia hacia sus semejantes en formación con el afán de mantener un estándar profesional y de llegar al reconocimiento que tanto ansían, en el contexto de un mercado competitivo y voraz, y de un ejercicio en conflictos de roles multifactoriales. Se podría afirmar que los enfermeros (varones) estarían menos inmiscuidos en situaciones de violencia hacia los estudiantes de enfermería, al no ubicarse en desniveles de poder relativos al género, respecto a otras profesiones predominantemente masculinizadas.

Desde un punto de vista pedagógico, las emociones generadas por ambientes propicios para el aprendizaje alcanzan notabilidad al definir espacios de acciones posibles de realizar. Estas emociones fueron descritas por Ibáñez [9], quien les asignó un espacio de relevancia, como facilitadores del aprendizaje: 'Emociones positivas o gratas permitirán la realización de acciones favorables para el aprendizaje. Emociones negativas o no gratas no lo permitirán'. Refiere también cómo los ambientes propicios producen que el estudiante manifieste mayor interés, pida ejemplos nuevos o realice más consultas, que sea capaz de profundizar conceptos, etc., lo cual favorece la construcción de su aprendizaje. Por el contrario, las situaciones que estudiantes chilenos de educación superior identifican como generadoras de emociones desfavorables para el aprendizaje, son ciertos aspectos metodológicos, incumplimiento de objetivos, de contenidos y de relaciones con sus docentes (entiéndanse las emociones desfavorables para el aprendizaje algunas tales como: rabia, impotencia, inseguridad, miedo, aburrimiento, desagrado y decepción). Entre las situaciones adversas para el aprendizaje, sobre aspectos relacionales, detallan: cuando el profesor o profesora 'es arbitrario, injusto, barrero', 'impone su opinión', 'no acepta la crítica ni otros puntos de vista', 'es autoritario, prepotente, inflexible, 'infunde temor, amenaza', 'abusa de su condición, se aprovecha de su autoridad', 'no permite opinar, no deja participar', 'se molesta al pedirle que explique de nuevo' o 'no se interesa en que aprendamos. 
A las situaciones cotidianas de la formación de los semejantes se suman las tensiones propias de la crisis de la enfermería en Chile. Además de haberse perfilado con reconocimiento en el interior de las instituciones, tiene un alto nivel en Latinoamérica. Sin embargo, debido a múltiples cambios en el escenario demográfico y epidemiológico, la población necesitada de cuidados ha crecido desproporcionadamente en relación con la existencia de enfermeras(os), lo que ha desencadenado que otros profesionales deban asumir funciones propias de su rol. Estos mismos conflictos de roles se transmiten a los estudiantes de enfermería, a través de la tensión generada en el interior de los equipos de salud. Aspectos tan relevantes que condicionan al estudiante como blanco de la violencia, son los señalados ciertamente por Letelier y Valenzuela: 'Por su condición de alumnos, son vulnerables a situaciones de abuso de poder... o son señalados como responsables de determinados actos que no han cometido, o cuando se les llama la atención delante del enfermo o de sus semejantes, o del resto del equipo de salud, avergonzándolos, menoscabando su autoimagen, olvidándose de la privacidad en que se debe evaluar o sugerir modificaciones de conducta, de modo de no estigmatizarlo, pero sí de guiarlo en su camino al aprendizaje... Existe, por tanto, una multiplicidad de factores de riesgo para la víctima, en este caso el alumno o alumna de enfermería, siendo de vital importancia su juventud, inexperiencia, impulsividad, imprudencia, poca habilidad, lentitud, dependencia de la docente $y$, en definitiva, menor poder' [8].

\section{Similitud entre la violencia hacia los estudiantes y la intrafamiliar}

Álvarez y Olivarí [10] definen la violencia intrafamiliar como 'una forma de descontrol en la convivencia, la cual, al prolongarse en el tiempo, legitima el uso de la agresión como forma de relación al interior del sistema familiar'. En concordancia con lo anterior, se presentan diversas formas en las que se visualiza la convivencia como algo descontrolado y donde la agresión es válida en las relaciones humanas. Muchas de ellas las encontramos también en las situaciones que viven los estudiantes durante su formación profesional: negligencia, al no dedicarle el tiempo y las atenciones suficientes para disminuir sus ansiedades y miedos, reafirmar conocimientos y adquirir destrezas; maltrato emocional, al ser blanco inofensivo de un sistema tan vertical, compartimentado y jerárquico como una institución sanitaria; potencial acoso moral, estando dentro de las posibilidades como objeto de descarga de autoritarismos, inseguridades, narcisismos, frustraciones; maltrato institucional, entendido como aquél que se ha generado en función de cómo se han definido oficialmente los procesos comunicativos y asistenciales, de acceso a la información y también por la complicidad institucional.

Las situaciones de violencia vienen dadas por la convergencia entre perfiles de personas maltratadoras y otras abusadas: unas que no reconocen sus características intrapersonales y sus consecuencias, y otras que tienden a asumir con facilidad unas culpas que no les pertenecen. Así, los actores se ven envueltos en un círculo en el cual crean una batería de símbolos comunicativos que pueden llegar a ser imperceptibles por quienes se encuentran fuera de esa espiral de violencia.

Si establecemos un paralelismo entre estos roles y los adoptados durante el ejercicio de la violencia en espacios formadores de enfermeros(as), la analogía es categórica. Un profesional de enfermería es, potencialmente, una persona maltratadora de estudiantes (concreta o simbólicamente). Este último puede entrar automáticamente en el círculo de violencia cuando cumple con el perfil (baja autoestima, dificultad para relacionarse, historia de maltratos previos, procedencia de familia con estudios incompletos); de no ser así, presencia el acto violento hacia su persona, pero realiza un ejercicio consciente de exclusión. En el ámbito de la formación destaca la presencia de testigos pasivos, quedando en evidencia un gran vacío. Este vacío correspondería a las falencias del sistema universitario para ofrecer al estudiante los canales activos de formalización de las denuncias, como una instancia formal y sólida de apoyo a las víctimas de maltrato. En ambas realidades, el modelo relacional parece perpetuarse en el tiempo. Enfermeras, maduras ya, informan haber sido blanco de situaciones injustas y violentas durante su proceso de formación, y se muestran en desacuerdo con los beneficios ofrecidos hoy en día al estudiante. 


\section{Conclusiones}

Queda en evidencia que toda la formación de los estudiantes de enfermería está grabada por la disciplina (rígida y constrictora), el control de los cuerpos en el sentido del aumento de su productividad y satisfacción del mercado.

Son las propias enfermeras las que realizan actos de violencia concreta o simbólica en pro de fomentar la productividad y de obtener reconocimiento. Su relación violenta con los estudiantes es análoga a los círculos de la violencia intrafamiliar, donde hay reproducción de roles y perpetuidad del modelo relacional. A través de esta forma de relacionarse, y al contrario de lo que desean, generan sentimientos adversos para el aprendizaje, provocando retraimiento, disminución de las consultas, disminución de la interacción con sus formadores y alejamiento del interés.

La violencia recibida en estos espacios de formación, un currículum rígido y horarios sobrecargados se suman a los actos violentos recibidos fuera del ambiente educacional, lo que hace que estos estudiantes tengan una calidad de vida deteriorada y se ponga en riesgo la humanización de la atención que en el futuro vayan a brindar.

Por lo pronto, es cardinal que la disciplina de enfermería mejore su cuerpo de conocimientos y su praxis en un marco conceptual que fortalezca la autoestima profesional de sus miembros y potencie la visibilidad de su aporte a la calidad de vida de las personas. Asimismo, es preciso que las universidades vigoricen la docencia clínica mediante la formación de tutores centrados en la psicología del aprendizaje.

\section{Bibliografía}

1. Ledy S, Pepper JM. Bases conceptuales de la enfermería profesional. New York: Organización Panamericana de la Salud; 1985.

2. Roberts S. Opressed group behavior: implications for nursing. Adv Nurs 1983; 5: 25-30.

3. Foucault M. Microfísica del poder. Madrid: Ediciones de La Piqueta; 1991.

4. Deleuze G. Conversaciones 1972-1990. Valencia: Pretexto; 1995.

5. Foucault M. Vigilar y castigar. El nacimiento de la prisión. Madrid: Siglo XXI; 1975. p. 314, 141, 156.

6. Edwards E. Cine para reflexionar. Revista Iberoamericana de Educación 2005; 37: 155-72.

7. Bourdieu P. ¿Qué significa hablar? La economía de los intercambios lingüísticos. Madrid: Akal; 1982.

8. Letelier P, Valenzuela S. Violencia: fenómeno relevante de estudio en campos clínicos intra-hospitalarios de enfermería. Ciencia y Enfermería 2002; 8: 21-6.

9. Ibáñez N. Las emociones en el aula. Estudios Pedagógicos 2002; 28: 31-45.

10. Álvarez P, Olivarí C. La terapia coactiva del maltrato infantil: la rehabilitación familiar en el contexto judicial. Psykhe 1993; 2: 53-7. 